\title{
Prevalence and clinical potential of extraspinal incidental findings in lumbosacral spine MRI of patients with suspected disc diseases
}

Sameeah Abdulrahman Rashid

pISSN: 0853-1773 • elSSN: 2252-8083 https://doi.org/10.13181/mji.oa.215550 Med J Indones. 2021;30:256-63

Received: May 28, 2021

Accepted: December 07, 2021

Authors' affiliations:

Department of Surgery, College of Medicine, Hawler Medical University, Erbil, Iraq

\section{Corresponding author:}

Sameeah Abdulrahman Rashid Department of Surgery, College of Medicine, Hawler Medical University, Kurdistan Region, Khanzad Street, Near Hawler Teaching Hospital. P.O. Box 40/0112, Erbil, Iraq

Tel/Fax: +964-66-222-7275

E-mail: sameeah.abdulrahman@hmu. edu.krd

\begin{abstract}
BACKGROUND Incidental findings could be observed in organs close to the spine while reporting lumbosacral spine magnetic resonance imaging (MRI). This study aimed to report the prevalence and clinical potential of extraspinal incidental findings in lumbosacral MRI of patients with suspected disc diseases.
\end{abstract}

METHODS This single-centered cross-sectional study was carried out on 420 consecutive adult patients who underwent lumbar spine MRI for suspected disc disease from January to July 2019. The MRI was checked for the presence of extraspinal incidental findings, and each finding was categorized according to the body organ and its clinical significance. Each MRI plane that best displayed the findings was recorded, and the association between the findings and patient's age and sex was determined.

RESULTS Of 420 samples, 135 cases showed extraspinal findings (32.1\%), and 7.6\% of the patients displayed suspicious lesions. The urinary tract was the most common system (18.6\%) to display both clinically significant (5.0\%) and benign findings (13.6\%), and the axial MRI section was the plane which showed most of the incidental findings. Incidental findings in any body system were rarely found in the younger patients. Females had significantly higher benign findings than males $(p=0.002)$.

CONCLUSIONS The prevalence of extraspinal findings in lumbosacral spine MRI is high, and some are significant. Most findings are related to the urinary tract and best displayed in the axial plane.

KEYWORDS incidental finding, lumbar spine, MRI
Incidental findings are observations discovered while conducting a test or procedure unrelated to the clinical indication or the primary aim of that particular test, ${ }^{1-4}$ and may have a potential clinical relevance. ${ }^{5}$ The management of incidental findings is still under debate, and the American College of Radiology has released a white paper in managing incidental findings on the abdominal computed tomography (CT). ${ }^{1}$ Many factors have contributed to the increased frequency of detecting incidental findings in medical imaging, such as the increased use of medical imaging, advancement in radiological viewing technology, 
introduction of picture archiving and communication systems into radiology practice, and improved image quality and resolution. ${ }^{1,2,4,6}$ Observing and reporting these findings raises various ethical and practical issues on clinical management, ${ }^{5,7}$ and debate is still existing on how to disclose incidental findings in the radiology report. Some incidental findings are benign with no clinical consequences; others may be more important than the suspected disease that prompted imaging of that particular region. ${ }^{3}$ Detection of clinically significant incidental findings could reduce morbidity and mortality when diagnosed earlier and managed in a timely manner. ${ }^{2,8}$

Many abdominopelvic organs are located near the lumbosacral spine region; hence, incidental lesions are likely to be observed within the field of view (FOV). This study aimed to report the prevalence and clinical potential of extraspinal incidental findings in lumbosacral spine magnetic resonance imaging (MRI) of patients with suspected disc diseases. We also aimed to determine the most common organ with incidental findings, the most common MR plane likely to detect the extraspinal incidental findings, and any possible association between the extraspinal incidental findings and patients' age and sex.

\section{METHODS}

This retrospective cross-sectional study was conducted on patients who underwent lumbosacral spine MRI in the Department of Radiology, PAR Private Hospital, Erbil, Iraq due to clinically suspected degenerative disc diseases from January to July 2019. Patients who were $<15$ years old, had MRI findings and/or history of recent trauma, had known primary spinal or extraspinal tumor, had secondary deposits, and had spinal infections were excluded from the study to produce more accurate estimates of the incidental findings in the healthy group. Patients with improper image quality due to movement artifacts and who had re-scanning during the study period were also excluded. Of 507 lumbar spine MRIs performed within the study period, only 420 cases were enrolled for further analysis. Approval from the research ethics committee of the College of Medicine, Hawler Medical University (Paper code: 7, approval date 22/6/2018) was obtained.

\section{MR imaging technique}

All lumbosacral spine MRIs were obtained with a 1.5T scanner (Optima; GE Medical system, USA) using a spine matrix coil integrated into the patient table. The protocols used for subjects with suspected lumbosacral spine disc disease were: sagittal $\mathrm{T}_{1}$ slice thickness of $3.5 \mathrm{~mm}$, intersection gap 1, matrix size $(320 \times 192)$, FOV $(34 \times 27.5) \mathrm{cm}$, repetition time (TR)/echo time (TE) (415/10.5 msec); sagittal T2 and sagittal $T_{2}$ fat-saturated sequence with a slice thickness of $3.5 \mathrm{~mm}$, intersection gap 1, matrix size $(380 \times 224)$, FOV $(34 \times 27.5) \mathrm{cm}$, TR/TE $(2700 / 107$ $\mathrm{msec})$; and selected axial T2 image through the discs with a slice thickness of $3 \mathrm{~mm}$, intersection gap 1, matrix size $(320 \times 160)$, FOV $(23 \times 18.5) \mathrm{cm}, \mathrm{TR} / \mathrm{TE}$ (2570/71.5 msec).

\section{Assessment and analysis of incidental findings}

All MR scans were evaluated for the presence of extraspinal incidental findings by a general radiologist with 16 years of experience. Evaluation continued by consulting with a more experienced radiologist when there was any doubt on the findings. The extraspinal incidental findings were categorized according to the body organ involved and further subcategorized as: (1) clinically insignificant findings; and (2) clinically significant findings. The clinically insignificant findings included normal anatomical variants or benign appearing lesions based on the characteristics of MR appearance or after a discussion with the relevant clinician. The clinically significant findings included (a) findings with MR appearance characteristics of significant diseases such as aortic aneurysm or hydronephrosis, (b) indeterminate lesions requiring further assessment by clinical/lab correlation, or (c) findings requiring further imaging studies or histopathological or surgical confirmation after a discussion with the relevant physician, such as renal or adrenal masses. The MR plane which best displayed the extraspinal incidental findings was documented. Age and sex of the patients were also recorded.

\section{Statistical analysis}

Data were analyzed using SPSS version 22 (IBM Corp., USA). Chi-square test of association was used to compare proportions. Fisher's exact test was used when more than $20 \%$ of the cells had an expected frequency of less than 5. A $p$-value of $\leq 0.05$ was considered statistically significant. 


\section{RESULTS}

The total number of patients enrolled was 420 , ranging from 15 to 86 years old. The mean (standard deviation) age was 47.73 (15.28) years old, and the median was 46 years old. Of all patients enrolled, $11.7 \%$ and $10.0 \%$ were aged $<30$ and $\geq 70$ years, respectively. Females constituted $55.7 \%$ of the patients. The basic characteristics of the subjects are shown in Table 1.

The prevalence of extraspinal incidental findings was $32.1 \%$ (135 cases out of 420 ). The detailed distribution of each specific extraspinal incidental findings, either benign or suspicious, based on its body system or organ of origin is presented in Table 2. Around one quarter (24.5\%) of the patients had benign findings (in any body system), and $7.6 \%$ had suspicious or significant findings. The proportion of females with benign findings (30.8\%) was significantly higher than males $(16.7 \%, p=0.002)$ as presented in Table 3.

Urinary tract had the most common incidental findings and the highest percentage of benign and suspicious findings among other systems (Table 2 and 3). The female genital tract was the next system to show the highest prevalence of incidental findings, as seen in Table 2. Benign findings were found in $19.2 \%$ of the females, while only $2.6 \%$ had suspicious findings (Table 3). Figure 1 shows the MR images of urinary tract findings (Figure 1a) and female genital tract findings (Figure $1 b$ ), which were incidentally discovered during lumbosacral spine MRI evaluation for suspected disc disease.

Table 3 shows that extraspinal incidental findings in any body system were rarely found in the younger

Table 1. Distribution of samples by age and sex

\begin{tabular}{ll}
\hline Characteristics & $\mathrm{n}(\%), \mathrm{N}=420$ \\
\hline $\begin{array}{l}\text { Age (years) } \\
<30\end{array}$ & $49(11.7)$ \\
$30-39$ & $94(22.4)$ \\
$40-49$ & $87(20.7)$ \\
$50-59$ & $80(19.0)$ \\
$60-69$ & $68(16.2)$ \\
$\geq 70$ & $42(10.0)$ \\
Sex & \\
\hline Male & $186(44.3)$ \\
\hline Female & $234(55.7)$ \\
\hline
\end{tabular}

Table 2. Types and distribution of incidental findings according to organs and systems

\begin{tabular}{|c|c|}
\hline Types of incidental findings & $\mathrm{n}(\%)$ \\
\hline \multicolumn{2}{|l|}{ Urinary system $(n=78)$} \\
\hline Simple renal cyst & $51(65.4)$ \\
\hline Hydronephrosis & $6(7.7)$ \\
\hline Prostate abnormality & $5(6.4)$ \\
\hline $\begin{array}{l}\text { Simple renal cyst \& urinary bladder } \\
\text { diverticulum or irregularity of the bladder wall } \\
\& \text { prostate abnormality }\end{array}$ & $4(5.1)$ \\
\hline $\begin{array}{l}\text { Urinary bladder diverticulum or irregularity of } \\
\text { the bladder wall }\end{array}$ & $3(3.8)$ \\
\hline Masses (renal, supra-renal, or bladder) & $3(3.8)$ \\
\hline $\begin{array}{l}\text { Absent one kidney, unilateral or bilateral small } \\
\text { kidney }\end{array}$ & $2(2.6)$ \\
\hline Horseshoe kidney & $1(1.3)$ \\
\hline $\begin{array}{l}\text { Simple renal cyst \& urinary bladder } \\
\text { diverticulum or irregularity of the bladder wall }\end{array}$ & $1(1.3)$ \\
\hline Simple renal cyst \& prostate abnormality & $1(1.3)$ \\
\hline $\begin{array}{l}\text { Urinary bladder diverticulum or irregularity of } \\
\text { the bladder wall \& prostate abnormality }\end{array}$ & $1(1.3)$ \\
\hline \multicolumn{2}{|l|}{ Genital tract $(n=51)$} \\
\hline Uterine fibroid & $18(35.2)$ \\
\hline Nabothian cyst & $10(19.6)$ \\
\hline Adenomyosis & $7(13.7)$ \\
\hline Simple ovarian cyst & $4(7.8)$ \\
\hline Suspicious endometrial lesion & $2(3.9)$ \\
\hline Absent uterus & $1(2.0)$ \\
\hline Hydrosalpinx & $1(2.0)$ \\
\hline Uterine fibroid \& nabothian cyst & $2(3.9)$ \\
\hline Uterine fibroid \& complex ovarian lesion & $1(2.0)$ \\
\hline Uterine fibroid \& post-partum changes & $1(2.0)$ \\
\hline Adenomyosis \& simple ovarian cyst & $1(2.0)$ \\
\hline Nabothian \& simple ovarian cysts & $1(2.0)$ \\
\hline Nabothian cyst \& complex ovarian lesion & $2(3.9)$ \\
\hline \multicolumn{2}{|l|}{ Lymphovascular system $(n=19)$} \\
\hline Atherosclerotic changes of the abdominal aorta & $10(52.6)$ \\
\hline Retroaortic renal vein & $5(26.3)$ \\
\hline Dilated abdominal aorta & $2(10.5)$ \\
\hline Dilated lymphatic channels & $1(5.3)$ \\
\hline Enlarged lymph nodes & $1(5.3)$ \\
\hline \multicolumn{2}{|l|}{ Gastrointestinal tract $(n=4)$} \\
\hline Thickened bowel wall & $3(75.0)$ \\
\hline Dilated common bile duct & $1(25.0)$ \\
\hline \multicolumn{2}{|l|}{ Others $(n=4)$} \\
\hline Peritoneal free fluid & $2(50.0)$ \\
\hline Abdominal wall mass & $1(25.0)$ \\
\hline Abdominal wall hernia & $1(25.0)$ \\
\hline
\end{tabular}


Table 3. Association of the incidental findings with sex and age

\begin{tabular}{|c|c|c|c|c|c|c|c|}
\hline \multirow{2}{*}{ Incidental findings } & \multicolumn{3}{|c|}{ Sex } & \multicolumn{4}{|c|}{ Age (years) } \\
\hline & Male, $\mathrm{n}(\%)$ & Female, n (\%) & $p$ & $<40, \mathrm{n}(\%)$ & $40-59, \mathrm{n}(\%)$ & $\geq 60, n(\%)$ & $p$ \\
\hline All systems & & & 0.002 & & & & $<0.001$ \\
\hline No findings & $142(76.3)$ & $143(61.1)$ & & $121(84.6)$ & $114(68.3)$ & $5(45.5)$ & \\
\hline Benign findings & $3(16.7)$ & $72(30.8)$ & & $19(13.3)$ & $44(26.3)$ & $40(36.4)$ & \\
\hline Significant findings & $13(7.0)$ & $19(8.1)$ & & $3(2.1)$ & $9(5.4)$ & $20(18.2)$ & \\
\hline Urinary system & & & 0.097 & & & & $<0.001$ \\
\hline No findings & $146(78.5)$ & $196(83.8)$ & & $139(97.2)$ & $145(86.8)$ & $58(52.7)$ & \\
\hline Benign findings & $26(14.0)$ & $31(13.2)$ & & $2(1.4)$ & $14(8.4)$ & $41(37.3)$ & \\
\hline Significant findings & $14(7.5)$ & $7(3.0)$ & & $2(1.4)$ & $8(4.8)$ & $11(10.0)$ & \\
\hline Genital tract & & & $<0.001 *$ & & & & $0.005^{*}$ \\
\hline No findings & $186(100.0)$ & $183(78.2)$ & & $128(89.5)$ & $138(82.6)$ & 103 (93.6) & \\
\hline Benign findings & $0(0)$ & $45(19.2)$ & & $14(9.8)$ & $27(16.2)$ & $4(3.6)$ & \\
\hline Significant findings & $0(0)$ & $6(2.6)$ & & $1(0.7)$ & $2(1.2)$ & $3(2.7)$ & \\
\hline Lymphovascular system & & & $0.551^{*}$ & & & & $0.001 *$ \\
\hline No findings & 178 (95.7) & $223(95.3)$ & & 141 (98.6) & $162(97.0)$ & $98(89.1)$ & \\
\hline Benign findings & $7(3.8)$ & $7(3.0)$ & & $2(1.4)$ & $5(3.0)$ & $7(6.4)$ & \\
\hline Significant findings & $1(0.5)$ & $4(1.7)$ & & $0(0)$ & $0(0)$ & $5(4.5)$ & \\
\hline Gastrointestinal tract & & & $0.133^{*}$ & & & & $0.005^{*}$ \\
\hline No findings & $186(100.0)$ & $230(98.3)$ & & $143(100.0)$ & $167(100.0)$ & $106(96.4)$ & \\
\hline Significant findings & $0(0)$ & $4(1.7)$ & & $0(0)$ & $0(0)$ & $4(3.6)$ & \\
\hline Others & & & $0.163^{*}$ & & & & $0.722 *$ \\
\hline No findings & $185(99.5)$ & 231 (98.7) & & 141 (98.6) & $165(98.8)$ & $110(100.0)$ & \\
\hline Benign findings & $1(0.5)$ & $0(0)$ & & $0(0)$ & $1(0.6)$ & $0(0)$ & \\
\hline Significant findings & $0(0)$ & $3(1.3)$ & & $2(1.4)$ & $1(0.6)$ & $0(0)$ & \\
\hline
\end{tabular}

*Fisher's exact test, $p$-value of $\leq 0.05$ was considered statistically significant

Figure 1. Extraspinal incidental findings in lumbar spine magnetic ressonance imaging (MRI) of two different patients. (a) Axial plane T2 weighted MRI of a middle-aged man showing severe hydronephrosis of the right kidney with diminished parenchymal thickness (white circle); (b) sagittal plane T2 weighted MRI of a young woman showing an anterior wall uterine corpus intramural fibroid (white circle)
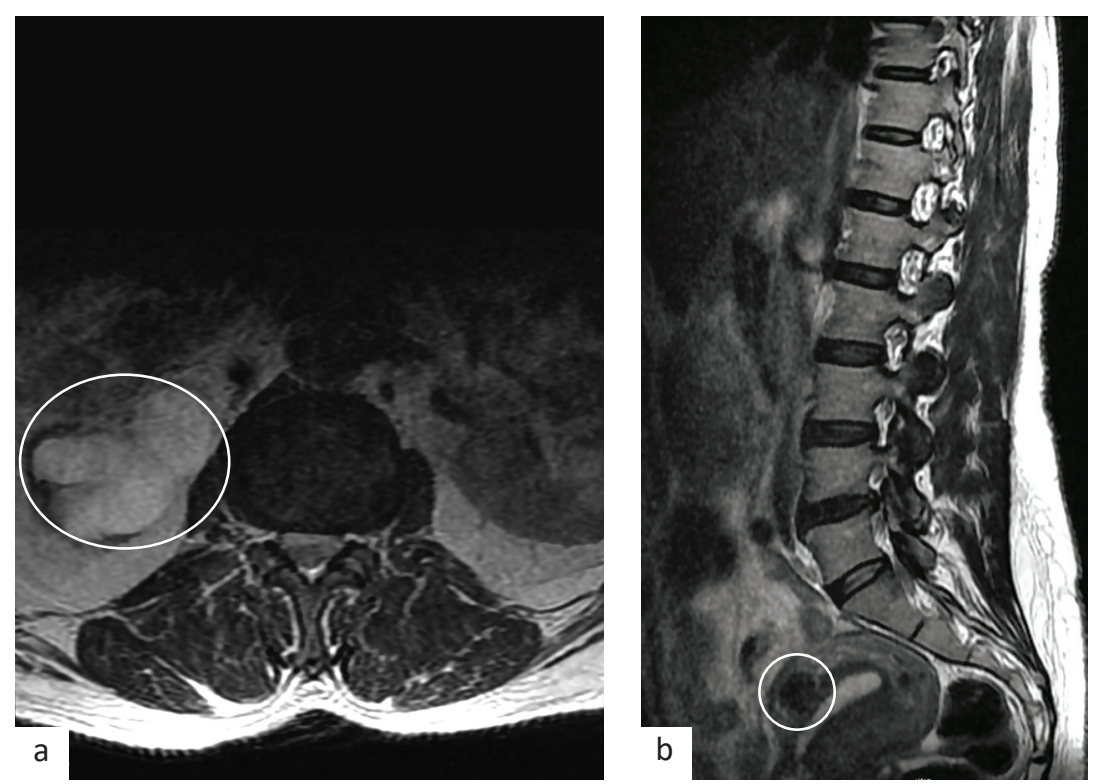
Table 4. The MRI plane used to diagnose the incidental lesions

\begin{tabular}{|c|c|c|c|c|}
\hline \multirow{2}{*}{ Type of incidental findings } & \multicolumn{4}{|c|}{ MRI plane } \\
\hline & Axial, n (\%) & Sagittal, n (\%) & Both, n (\%) & $p$ \\
\hline Urinary system & & & & $<0.001^{*}$ \\
\hline Benign finding & $50(87.7)$ & $5(8.8)$ & $2(3.5)$ & \\
\hline Need further evaluation & $8(38.1)$ & 9 (42.9) & $4(19.0)$ & \\
\hline Genital tract & & & & NA \\
\hline Need further evaluation & $0(0)$ & $4(66.7)$ & $2(33.3)$ & \\
\hline Lymphovascular & & & & $0.037^{*}$ \\
\hline Benign finding & $13(92.9)$ & $0(0)$ & $1(7.1)$ & \\
\hline Need further evaluation & $2(40.0)$ & $1(20.0)$ & $2(40.0)$ & \\
\hline Gastrointestinal & & & & NA \\
\hline Need further evaluation & $1(25.0)$ & $1(25.0)$ & $2(50.0)$ & \\
\hline Others & & & & $0.500^{*}$ \\
\hline Benign finding & $0(0)$ & $1(100.0)$ & $0(0)$ & \\
\hline Need further evaluation & $1(33.3)$ & $0(0)$ & $2(66.7)$ & \\
\hline
\end{tabular}

$\mathrm{MRI}=$ magnetic resonance imaging; $\mathrm{NA}=$ not applicable *Fisher's exact test age group, with $p<0.001$. Similarly, incidental findings in the lymphovascular system were commonly detected in the older age group, especially in the $\geq 60$ years old group $(p=0.001)$

Benign extraspinal incidental findings were detected in $37.3 \%$ of patients aged $\geq 60$ years, and significant extraspinal incidental findings were seen in $10.0 \%$ of the cases; meanwhile, other age groups had significantly lower percentages $(p<0.001)$. In contrast, the 40-59 years old group had the highest genital tract incidental findings $(p=0.005)$.

The axial plane was the most common plane to detect the lymphovascular incidental findings regardless of its significance $(p=0.037)$ (Table 4$)$. In the urinary system, the axial plane detected $87.7 \%$ of the benign findings and $38.1 \%$ of the clinically suspicious findings $(p<0.001)$. No significant association was detected between the MRI plane and genital tract findings $(p=0.186)$. The other findings are presented in Table 4.

\section{DISCUSSION}

The prevalence of extraspinal incidental findings in this study was $32.1 \%$, which is close to the result of Maxwell et al. ${ }^{9}$ However, our result was higher than Tuncel et $\mathrm{al}^{10}$ who found a low prevalence of extraspinal incidental findings due to the exclusion of cases with renal cysts, ovarian cysts, and fibroids measuring $1-1.5 \mathrm{~cm}$ size. Ibrahim et al ${ }^{11}$ also reported lower prevalence than our study, which is likely related to the lower mean age of their study sample. Meanwhile, a higher prevalence of incidental findings found by Lee et $\mathrm{al}^{8}$ was caused by the use of CT scans that has a high sensitivity for urinary stone detection. The high prevalence of extraspinal incidental findings should raise the awareness among radiologists and clinicians to carefully assess the extraspinal structures while evaluating lumbosacral spinal MRI to minimize missing findings and all its clinical consequences.

Considering the clinical potential of the extraspinal incidental findings, we observed that $7.6 \%$ of the examined patients had clinically significant findings, which constituted $23.7 \%$ of all the detected incidental findings. Although this result is similar to Ibrahim et al, ${ }^{11}$ Maxwell et al ${ }^{9}$ found higher clinically significant findings due to different samples' age (older than this study), the use of wider MRI FOV, and additional use of the coronal plane in the spine protocol. Early detection of the clinically significant lesion could reduce morbidity and mortality.

We have observed a statistically significant association between extraspinal incidental findings and age. The younger age groups had fewer extraspinal incidental findings compared with the higher age groups. The association between age and extraspinal incidental findings is in line with some previous studies. ${ }^{12-14}$ However, we found different results between age groups for the most incidental 
findings. The three previous studies showed that most extraspinal incidental findings were found in patients aged $<60$ years. In contrast, we found only $15.4 \%$ of patients aged $<40$ years had extraspinal incidental findings. This difference is caused by more than $25 \%$ of our subjects were aged $>60$. Meanwhile, Qasim et $\mathrm{al}^{13}$ and Sobhan et $\mathrm{al}^{14}$ had much lower proportion of cases aged $\geq 60$ years.

Considering the association between age and particular organ incidental findings, older patients showed more extraspinal incidental findings in the urinary and lymphovascular systems, while younger patients were more likely to have findings in the female genital tract. This result agrees with Qasim et $\mathrm{al}^{13}$ who observed a similar pattern. Our result is supported by an increased incidence of simple renal cysts with age. ${ }^{15}$ Meanwhile, the cumulative incidence of uterine leiomyoma tends to increase from the age of 35 to 50 years and slow down at older age..$^{16}$

In the present study, a significant association was observed between the presence of extraspinal incidental findings and sex. Similar to Zidan et al ${ }^{12}$ and Qasim et al, ${ }^{13}$ most extraspinal incidental findings in this study were found in females. In contrast, Sobhan et al ${ }^{14}$ showed no such association as they excluded any extraspinal incidental findings related to genital tracts, such as uterus, ovary, or prostate.

We found that the urinary tract has the highest number of incidental findings and the highest percentage of benign (simple renal cyst) and suspicious findings (hydronephrosis) among all body systems, followed by the female genital tract. Our result agrees with many previous studies.6,10,12,14 This is because the urinary tract is affected by many pathologies such as renal cyst, stone/ hydronephrosis, and bladder changes secondary to prostate enlargement, especially with advancing age; and the female genital tract can be affected by simple ovarian cyst and uterine fibroids.

Simple renal cyst becomes more prevalent in older age because of progressive nephron loss with aging, ${ }^{12}$ confirming that the simple renal cyst formed the largest proportion of the detected extraspinal incidental findings. Although the etiology for hydronephrosis may not be recognized in lumbosacral spinal MRI because of the limited study protocol, the detection of this finding is important to prevent irreversible damage to the affected kidney and address potential problems to the other kidney, especially when observed early., ${ }^{7,17}$

Other significant findings in the urinary system in our study were few cases with either renal, adrenal or bladder masses, unilateral small or absent kidney, and horseshoe kidney. This result is in line with previous studies; 8,12 however, Tuncel et $\mathrm{al}^{10}$ found 11 adrenal masses as their axial MR sections were captured at the upper lumbar level descending from L1 vertebra, which likely ran through the adrenal gland level and resulted in a clearer view of the adrenal gland. Detection of these masses could increase survival rate and improve prognosis, since the incidental detection of renal cell carcinoma would carry better prognosis than tumors presenting with symptoms, mostly due to lower tumor stage at time of diagnosis. ${ }^{17}$

Of 19 patients with incidental findings in the lymphovascular system, five were clinically significant, including dilated abdominal aorta or enlarged lymph nodes. Although this result is different from other previous studies, ${ }^{7,10,11}$ all studies including our study found a similar result of a lower incidental findings frequency in the lymphovascular than in the urinary and genital tracts. These difference may be caused by the age, ethnicity, technique used such as FOV, and use of signal saturation anterior to the spine, thereby limiting proper retroperitoneum evaluation (aorta and the nearby lymph nodes). Aortic aneurysm could have serious clinical consequences, mainly from the risk of rupture, and enlarged lymph nodes may signal the presence of primary lymphoproliferative disease or deposits from a hidden primary. ${ }^{7}$ The detection of these incidental findings could help in saving patients' life and reducing mortality.

We have observed that axial plane was the most common plane to detect extraspinal incidental findings within the lymphovascular and urinary systems. In the urinary tract, the axial plane could show $87.7 \%$ of the benign findings and $38.1 \%$ of the suspicious incidental findings, while the sagittal plane displayed most of the incidental findings related to female genital tract. This observation is related to the location of organs and scanning where the uterus, cervix, and vagina are midline structures and best viewed in the sagittal section. Axial sections for lumbosacral spinal MRI are usually taken through the selected lumbar intervertebral discs level, which is a section higher than uterine level and less likely to run 
through the female genital organ. Adrenal glands, kidneys, and ureters are located paracentrally, and the sagittal plane for lumbosacral spinal MRI may not run through them; therefore, incidental findings related to these structures are most often seen in the axial images. ${ }^{17}$

Lumbosacral spine MRI, as one of the commonly performed study in the Department of Radiology, involves radiologists from different levels to interpret and report the scan and practitioners from other specialties to evaluate the scan based on their expertise. The high prevalence of extraspinal incidental findings with a non-ignorable percentage of suspicious extraspinal incidental findings in the lumbosacral spinal MRI should raise the awareness of radiologists and other involved specialties in improving clinical practice and considering a structured evaluation of all extraspinal structures within the scanned field, thus avoid missing a significant finding that affects the patient care and medicolegal consequence.

Many worldwide screening programs are aimed to detect diseases at an early stage when treatment may be more effective..$^{18}$ The role of an appropriate evaluation of lumbosacral spinal MRI in detecting potentially significant or life-threatening incidental findings, such as hydronephrosis, aortic aneurysm, or tumor, is higher than relying on the available screening tools in increasing patient survival rate and improving patient outcome.

This study has some limitations. The data collection was obtained from a single center; hence, the result may not be generalized. Surgical confirmation and/or follow-up was not achieved for every case with clinically significant incidental findings. The diagnosis of some cases was based on the observations from lumbosacral spinal MRI, so the actual prevalence of these findings may be inaccurate. Limitations of lumbosacral spinal MRI in finding extraspinal incidental findings included the use of limited sequences and planes and its narrow FOV, especially with the use of saturation bands.

In conclusion, the prevalence of extraspinal incidental findings in lumbosacral spinal MRI is high and clinically significant in some cases, with a significant correlation to age and sex. The urinary system displayed the highest percentage of the incidental findings, and the axial MR section was the most common plane to display the incidental findings, particularly for the urinary system. A structured and proper review of all extraspinal anatomical organs within the scanned field is recommended.

\section{Conflict of Interest}

The authors affirm no conflict of interest in this study.

\section{Acknowledgment}

We would like to thank the Radiology staffs of PAR Hospital.

\section{Funding Sources}

None.

\section{REFERENCES}

1. Berland LL, Silverman SG, Gore RM, Mayo-Smith WW, Megibow AJ, Yee J, et al. Managing incidental findings on abdominal CT: white paper of the ACR incidental findings committee. J Am Coll Radiol. 2010;7(10):754-73.

2. Ells $C$, Thombs BD. The ethics of how to manage incidental findings. CMAJ. 2014;186(9):655-6.

3. Park HJ, Jeon YH, Rho MH, Lee EJ, Park NH, Park SI, et al. Incidental findings of the lumbar spine at MRI during herniated intervertebral disk disease evaluation. Am J Roentgenol. 2011;196(5):1151-5.

4. Varghese B, Babu AC, Kumar A, Priya PS, John JS, Noufal M, et al. Cross-sectional study on incidental spinal findings in magnetic resonance imaging lumbar spine of patients with low back pain. West Afr J Radiol. 2017;24(1):38-44.

5. Vernooij MW, Ikram MA, Tanghe HL, Vincent AJ, Hofman A, Krestin GP, et al. Incidental findings on brain MRI in the general population. N Engl J Med. 2007;357(18):1821-8.

6. Wagner SC, Morrison WB, Carrino JA, Schweitzer ME, Nothnagel $\mathrm{H}$. Picture archiving and communication system: effect on reporting of incidental findings. Radiology. 2002;225(2):500-5.

7. Quattrocchi CC, Giona A, Di Martino AC, Errante Y, Scarciolla L, Mallio CA, et al. Extra-spinal incidental findings at lumbar spine $\mathrm{MRI}$ in the general population: a large cohort study. Insights Imaging. 2013;4(3):301-8.

8. Lee SY, Landis MS, Ross IG, Goela A, Leung AE. Extraspinal findings at lumbar spine $\mathrm{CT}$ examinations: prevalence and clinical importance. Radiology. 2012;263(2):502-9.

9. Maxwell AW, Keating DP, Nickerson JP. Incidental abdominopelvic findings on expanded field-of-view lumbar spinal MRI: frequency, clinical importance, and concordance in interpretation by neuroimaging and body imaging radiologists. Clin Radiol. 2015;70(2):161-7.

10. Tuncel SA, Çaglı B, Tekataş A, Kırıcı MY, Ünlü E, Gençhellaç H. Extraspinal incidental findings on routine MRI of lumbar spine: prevalence and reporting rates in 1278 patients. Korean J Radiol. 2015;16(4):866-73.

11. Ibrahim $\mathrm{H}$, Elsadawy $\mathrm{ME}$. Incidental findings in lumbar spine MRI: their prevalence and potential impact on patient management. Egypt J Radiol Nucl Med. 2019;50(59).

12. Zidan MMA, Hassan IA, Elnour AM, Ali WM, Mahmoud MZ, Alonazi $B$, et al. Incidental extraspinal findings in the lumbar spine during magnetic resonance imaging of intervertebral discs. Heliyon. 2018;4(9):e00803.

13. Qasim HA, Al-Bayati H, Mahmoud NA. Incidental finding during MRI evaluation of lumbosacral disc prolapse in adults in Al-Hilla Teaching Hospital. Med J Babylon. 2017;14(3):53749.

14. Sobhan M, Samiee M, Asgari Y, Ahmadi M. Incidental findings of the lumbar spine at MRI in patients diagnosed with discopathy. Int J Med Imaging. 2016;4(5):44-7.

15. Ozveren B, Onganer E, Türkeri LN. Simple renal cysts: 
prevalence, associated risk factors and follow-up in a health screening cohort. Urol J. 2016;13(1):2569-75.

16. Stewart EA, Cookson CL, Gandolfo RA, Schulze-Rath R. Epidemiology of uterine fibroids: a systematic review. BJOG. 2017;124(10):1501-12.
17. Gebara NV, Meltzer DE. Extraspinal findings on lumbar spine MR imaging. J Radiol Case Rep. 2009;3(8):5-13.

18. Wilken D, Baur X, Barbinova L, Preisser A, Meijer E, Rooyackers $J$, et al. What are the benefits of medical screening and surveillance? Eur Respir Rev. 2012;21(124):105-111. 